\title{
Scherbengericht
}

Liebe Leserinnen, liebe Leser,

Vertrauen ist Alltag, auch bezüglich akademischer Grade. Eine Doktorarbeit qualifiziert ihren Autor als eigenständigen wissenschaftlichen Denker. Die Universität bescheinigt es; ihr vertraut man. So hält man es seit Jahrhunderten. Aber man nimmt es heute nicht mehr so genau. Es gibt Fachgebiete, in denen die Doktorarbeit nicht der Beginn eines Forscherlebens sondern nur der Abschluss einer herkömmlichen Berufsausbildung ist, neben anderen, wo neue, relevante und authentische Erkenntnisse erwartet werden.

Vor einiger Zeit stellte ein Doktorand beim Quellenstudium fest, dass einer seiner Vorgänger, ein bekannter Politiker, aus anderen Arbeiten abgeschrieben hatte; es kam an die Öffentlichkeit. Die Universität, die den Titel vergeben hatte, prüfte den Fall, erkannte auf Plagiate und entzog dem Plagiator den Titel. Damit machte sie sich um die Wissenschaft verdient. Die öffentliche Meinung zeigte sich zufrieden und übersah den Schuldanteil der Universität, die offensichtlich den Doktoranden nicht ausreichend wissenschaftlich begleitet und die Plagiate übersehen hatte. Die Aufdeckung machte Schule und führte zu drei analogen Fällen bekannter Politiker. In einem davon bestätigte sich der Verdacht nicht; der Doktor hatte nicht abgeschrieben. Aber - gängige Volksweisheit - einmal in das unscharfe Gedächtnis der Öffentlichkeit geraten, bleibt von einem Verdacht immer etwas haften. Was immer Abträgliches in die Medien gerät, kann rufschädigend sein.

An der durch diese Fälle entfachten öffentlichen Meinung fällt auf, dass von den beiden Schuldträgern - Doktorand und Universität - die Institution besser wegkommt als das Individuum; sie wird lustriert, das Individuum einem Scherbengericht unterzogen. Weil Doktorarbeiten in der Regel veröffentlicht sind, war es konsequent, Plagiate an die Öffentlichkeit zu bringen. Der Datenschutz zeigte sich davon wenig betroffen, da veröffentlichte Daten ihn wenig berühren; seine Beteiligung an der öffentlichen Diskussion war nicht geboten. Die allgemeine politisch korrekte Meinung hielt es mit Jean Jaques Rousseau: sie wollte Transparenz und bekam sie. Doch gibt der Fall des falsch Beschuldigten zu denken. Sein guter Ruf war beschädigt. Unschuldig muss er die Spannung zwischen gesellschaftlicher Transparenzforderung und individueller Privatheit aushalten.

Dem Rechtssystem stellt sich die Frage: Ist es für die Gesellschaft wichtiger, dass sie sich das Individuum transparent hält, oder soll sie es sich kooperativ halten, indem sie ihm eine Privatsphäre belässt? In dieser braucht es sich nicht gesellschaftlich korrekt zu verhalten, kann lügen, täuschen, heimlich sündigen. Außerhalb dieser Sphäre muss es die Forderung nach Transparenz bedienen. Die Grenzen zwischen Privatsphäre und Öffentlichkeit werden vom positiven Recht gezogen, die Einzelinteressen nivellierend. Je nach Großzügigkeit der Grenzziehung ist das Individuum nicht nur gegen Transparenzforderungen, sondern auch gegen die Ahndung von leichteren Rechtsverstößen geschützt. Im Sinne der Grundstimmung, die dem Datenschutz unterliegt, wird damit der gesellschaftliche Druck auf das Individuum abgemildert, ohne dass das Wohl der Gesellschaft dabei Schaden zu nehmen braucht. In diese von der Großzügigkeit geöffnete Lücke passt der Datenschutz. Er schützt es gegen die Zudringlichkeit der Gesellschaft; er ist, seinem Ziele nach, für das Wohl des Individuums voreingenommen.

Ich will jetzt nicht, liebe Leserinnen und Leser, die Frage stellen, ob nicht der Datenschutz auch den persönlichen Ruf eines verdächtigten Doktors besser schützen sollte. Vielmehr interessiert mich in diesem Zusammenhange die Frage: Was tut sich in der Grundstimmung der Gesellschaft? Wie alles ist auch sie im Fluss und ändert sich im Laufe der Generationen; sie hat sich seit den 70er-Jahren bereits deutlich verändert. Offensichtlich wächst - von der Technikentwicklung unterstützt und herausgefordert - die Geneigtheit zurTransparenz. Entsprechend nimmt der Respekt vor der Privatsphäre ab. Duldet doch die Grundstimmung, dass mittlerweile automatisiert nach Plagiaten gesucht wird und dass im Zweifelsfalle auch der Ruf eines Unschuldigen geschädigt werden darf.

Mit freundlichen Grüßen, Ihr

\section{Karl Rhavels}

